Proceeding Series of the Brazilian Society of Computational and Applied Mathematics

\title{
Um novo tipo de método de projeção com diferenças finitas compactas de alta ordem
}

\author{
Gabriela Aparecida dos Reis ${ }^{1}$ \\ Italo Valença Mariotti Tasso ${ }^{2}$ \\ Leandro Franco de Souza ${ }^{3}$ \\ José Alberto Cuminato ${ }^{4}$
}

Instituto de Ciências Matemática e de Computação - ICMC, USP, São Carlos, SP.

Resumo Neste trabalho é apresentado um novo tipo de método de projeção que possui quarta ordem de convergência espacial e segunda ordem temporal para a velocidade e pressão. Esse método é particularmente interessante, pois não requer o uso de aproximações para a pressão (na equação do momento) e nem condições de contorno complicadas para a velocidade intermediária. Além disso, trata-se de um método de projeção exata, onde a condição de incompressibilidade é satisfeita exatamente (a menos da precisão de máquina) a cada passo de tempo.

Palavras-chave. Navier-Stokes, método de projeção, diferenças finitas compactas, métodos de alta ordem.

\section{Introdução}

A dinâmica de um escoamento Newtoniano incompressível descrito pelas equações de Navier-Stokes é dada por

$$
\begin{aligned}
\frac{\partial \mathbf{u}}{\partial t}+\mathbf{u} \cdot \nabla \mathbf{u} & =-\nabla p+\nu \nabla^{2} \mathbf{u}+\mathbf{g}, \\
\nabla \cdot \mathbf{u} & =0
\end{aligned}
$$

onde $\nu$ é viscosidade cinemática, $\mathbf{u}=(u, v)$ o vetor velocidade, $p$ a pressão sobre densidade constante e g o campo gravitacional. As condições de contorno do tipo Dirichlet para a velocidade são $\left.\mathbf{u}\right|_{\partial \Omega}=\mathbf{u}_{b}$. Não há condições de contorno para a pressão quando as condições de contorno da velocidade são do tipo Dirichlet.

\footnotetext{
${ }^{1}$ gareis@icmc.usp.br

2italo@tasso.com.br

3 lefraso@icmc.usp.br

${ }^{4}$ jacumina@icmc.usp.br
} 


\section{Métodos de projeção}

Em geral, um método de projeção, com segunda ordem temporal, segue os seguintes passos [3]

Passo 1: Define-se um campo de velocidade intermediário $\mathbf{u}^{*}$ que pode ser calculado de

$$
\begin{aligned}
\frac{\mathbf{u}^{*}-\mathbf{u}^{n}}{\Delta t} & =-\nabla q-[\mathbf{u} \cdot \nabla \mathbf{u}]^{n+1 / 2}+\nu \nabla^{2} \mathbf{u}^{n+1 / 2}+\mathbf{g}^{n+1 / 2}, \\
B\left(\mathbf{u}^{*}\right) & =0
\end{aligned}
$$

onde $q$ representa uma aproximação para $p^{n+1 / 2}$ e $B\left(\mathbf{u}^{*}\right)=0$ condições de contorno para $\mathbf{u}^{*}$. Cada método especifica escolhas diferentes para $q$ e $B\left(\mathbf{u}^{*}\right)$. Essas escolhas influenciam significativamente a ordem temporal do método.

Passo 2: Faz-se a projeção

$$
\begin{aligned}
\mathbf{u}^{*} & =\mathbf{u}^{n+1}+\Delta t \nabla \phi^{n+1}, \\
\nabla \cdot \mathbf{u}^{n+1} & =0,
\end{aligned}
$$

usando condições de contorno consistentes com $B\left(\mathbf{u}^{*}\right)=0$ e $\left.\mathbf{u}^{n+1}\right|_{\partial \Omega}=\mathbf{u}_{b}^{n+1}$.

Esse sistema reduz-se a uma equação de Poisson quando se aplica o operador divergente a (3a)

$$
\nabla \cdot \nabla \phi^{n+1}=\frac{1}{\Delta t} \nabla \cdot \mathbf{u}^{*}
$$

com condições de contorno $\left.\hat{\mathbf{n}} \cdot \nabla \phi^{n+1}\right|_{\partial \Omega}=\hat{\mathbf{n}} \cdot\left(\left.\mathbf{u}^{*}\right|_{\partial \Omega}-\mathbf{u}_{b}^{n+1}\right)$.

Passo 3: Atualiza-se a pressão $p^{n+1 / 2}=q+L\left(\phi^{n+1}\right)$, onde a função $L$ representa a dependência de $p^{n+1 / 2}$ sobre $\phi^{n+1}$. Essa função depende do tipo de discretização temporal usada nos termos viscoso e convectivo. A nova velocidade $\mathbf{u}^{n+1}$ é calculada por (3a).

\subsection{Método de projeção com pressão incremental e livre de pressão}

A escolha da aproximação da pressão $q$, da condição de contorno $B\left(\mathbf{u}^{*}\right)$ e das aproximações temporais para os termos viscoso e convectivo são importantes na determinação do método. As condições de contorno para $\mathbf{u}^{*}$ devem ser consistentes com a equação (3a), embora essa dependa de valores de $\phi^{n+1}$ que ainda são desconhecidos e precisam ser aproximados.

Em [2] considera-se $q=p^{n-1 / 2}$ e $B\left(\mathbf{u}^{*}\right)=\left.\left(\mathbf{u}^{*}-\mathbf{u}_{b}^{n+1}\right)\right|_{\partial \Omega}=0$. A pressão é atualizada por $p^{n+1 / 2}=p^{n-1 / 2}+\phi^{n+1}$. Essa expressão de atualização da pressão é valida quando se utiliza métodos explícitos para os termos viscoso e convectivo. Se outros métodos não totalmente explícitos forem utilizados para os termos convectivo ou viscoso, mais termos apareceriam na equação da pressão.

Esse método é considerado como sendo um método de projeção com pressão incremental (do inglês incremental-pressure projection method). A ideia por trás dessa 
classe de métodos é que o termo $q$, que aproxima $p^{n+1 / 2}$, faça com que $\mathbf{u}^{*}$ fique mais próximo de $\mathbf{u}^{n+1}$, e com isso possa se usar as mesmas condições de contorno para ambos.

Por outro lado, em [4] considera-se $q=0$. Como condição de contorno para $\mathbf{u}^{*}$, usa-se $\left.\mathbf{u}^{*}\right|_{\partial \Omega}=\mathbf{u}_{b}^{n+1}+\left.\Delta t \nabla \phi^{n}\right|_{\partial \Omega}$. A pressão é dada por $p^{n+1 / 2}=\phi^{n+1}$, para discretizações temporais explícitas. Visto que não há pressão na equação do momento, esse tipo de método é chamado de método de projeção livre de pressão (do inglês pressure-free projection method). Isso é interessante pois erros no gradiente da pressão são eliminados da equação do momento, os quais podem se acumular com o tempo.

A falta de consistência na escolha de $q$ e $B\left(\mathbf{u}^{*}\right)$ ou no cálculo de $L\left(\phi^{n+1}\right)$ pode introduzir erros numéricos não somente nos transientes como também nos estados estacionários. Por exemplo, a escolha $q=0$ e $B\left(\mathbf{u}^{*}\right)=\left.\left(\mathbf{u}^{*}-\mathbf{u}_{b}^{n+1}\right)\right|_{\partial \Omega}=0$ pode introduzir um erro proporcional a $\Delta t$ nos transientes e no estado estacionário [7].

Além disso, é importante garantir que a cada passo de tempo a condição de incompressibilidade é satisfeita. A próxima seção descreve como isso pode ser garantido.

\subsection{Estêncil compatível: projeção exata e projeção aproximada}

O fato de um método de projeção ser exato ou aproximado, está relacionado com a resolução da equação de Poisson (4). Quando se resolve esta equação

$$
\nabla \cdot \nabla p=\frac{1}{\Delta t} \nabla \cdot \mathbf{u}^{*}
$$

especial atenção deve se ter com o operador $(\nabla \cdot \nabla)$. Enquanto que no caso contínuo esse operador é o mesmo que o laplaciano $\nabla^{2}$, no caso discreto isso não é sempre verdade. Em particular, quando se trata de diferenças finitas compactas, não é possível obter um operador laplaciano compacto que seja exatamente igual ao operador $(\nabla \cdot \nabla)$. Se um operador laplaciano compacto for usado em vez disso, o método de projeção não será exato, mas apenas aproximado, e a condição de incompressibilidade será satisfeita a menos da ordem do método [3].

\subsection{Projeção exata com diferenças finitas compactas}

Para obter um método exato com diferenças finitas compactas, a equação diferencial (4), que é bidimensional e de segunda ordem, pode ser reescrita como

$$
\begin{aligned}
& p_{x}-\frac{\partial}{\partial x} p=0, \\
& p_{y}-\frac{\partial}{\partial y} p=0, \\
& p_{x x}-\frac{\partial}{\partial x} p_{x}=0, \\
& p_{y y}-\frac{\partial}{\partial y} p_{y}=0, \\
& p_{x x}+p_{y y}=\frac{1}{\Delta t}\left(\frac{\partial u^{*}}{\partial x}+\frac{\partial v^{*}}{\partial y}\right),
\end{aligned}
$$


que é um sistema de equações diferenciais unidimensionais de primeira ordem, com exceção da última que é puramente algébrica, uma vez que o termo do lado direito pode ser calculado previamente. Desta forma, as diferenças finitas compactas podem ser facilmente aplicadas para discretizar cada equação. Se a mesma discretização for usada para todas as equações, um método de projeção exata será obtido.

Em malhas deslocadas, as quantidades $p, p_{x x}$ e $p_{y y}$ estão no centro das células, enquanto $p_{x}$ e $p_{y}$ estão sobre as arestas das células, na mesma posição que $u^{*}$ e $v^{*}$, respectivamente.

Quando se calcula $\mathbf{u}^{*}$, assume-se que $\mathbf{u}^{*}=\mathbf{u}_{b}^{n+1}$ no contorno, o que é equivalente a considerar $p_{x}=0$ e $p_{y}=0$ no contorno. Dessa forma, nem $u^{*}, v^{*}, p_{x}$ ou $p_{y}$ precisam ser calculados no contorno. Essa escolha de condições de contorno não compromete a precisão do método, mesmo no caso de projeção livre de pressão, como será mostrado na seção de resultados.

Essa abordagem tem várias vantagens:

- não é necessário construir estênceis para o operador laplaciano, o que é bastante complexo quando se quer um método exato baseado em diferenças finitas compactas. Este procedimento é, no entanto, possível, mas o estêncil resultante não seria compacto, e poderia se estender ao longo do comprimento do domínio. Um exemplo de como fazer isso pode ser encontrado em [1];

- além de calcular a pressão $p$, as quantidades $p_{x}$ e $p_{y}$ já são calculadas para o próximo passo do método de projeção, ou seja, a correção da velocidade;

- não são necessárias células fantasmas, interpolações ou extrapolações;

- cantos no domínio computacional não necessitam de tratamento especial, uma vez que apenas equações unidimensionais estão presentes.

A única desvantagem encontrada é que o sistema linear é maior em relação ao número de equações e incógnitas. No enteando, a matriz é esparsa quando comparada àquela usada em [1]. Na presente abordagem, cada linha da matriz tem, no máximo, 6 elementos não nulos, independentemente do tamanho do domínio. Em [1], o número de não zeros é proporcional ao comprimento do domínio. Desta forma, um domínio $n \times n$ produziria uma matriz com $\mathcal{O}\left(n^{2}\right)$ não zeros na presente formulação, enquanto que com [1] a matriz poderia ter $\mathcal{O}\left(n^{3}\right)$ não zeros.

\section{Resultados}

A seguir, três métodos de projeção com diferenças finitas compactas serão analisados: método de projeção livre de pressão e aproximado (LP-A), método de projeção com pressão incremental e aproximado (PI-A) e método de projeção livre de pressão e exato (LP-E). Todos utilizam diferenças finitas compactas de quarta ordem para aproximar as derivadas espaciais. O método de Adams-Bashfoth de dois passos (segunda ordem) é usado na discretização temporal [9]. As condições de contorno para a velocidade intermediaria $\mathbf{u}^{*}$ são as mesmas da velocidade $\mathbf{u}$. 
Esses métodos diferem em dois aspectos. Primeiro, os métodos LP-A e LP-E utilizam $q=0$ em (2a), enquanto o PI-A usa $q=p^{n-1 / 2}$. Segundo, os métodos LP-A e PI-A resolvem a equação de Poisson discretizando o operador laplaciano como em [5], enquanto o LP-E segue o que foi descrito na seção 2.3.

\subsection{Escoamento forçado em uma cavidade}

O problema clássico de escoamento laminar dentro de uma cavidade quadrada na qual a tampa se move é simulado. Uma solução analítica para o estado estacionário é conhecida e dada por [8]. O domínio é um quadrado unitário com condições de contorno do tipo Dirichlet.

Observa-se na figura 1a que no LP-A o refinamento da malha deixa de produzir ganho de precisão a partir de um certo nível de refinamento. Com a redução do $\Delta t$, figura 1b, há um ganho de precisão para malhas mais finas no estado estacionário. Isso evidencia que esse método introduz um erro proporcional ao $\Delta t$ e que esse erro está presente inclusive no estado estacionário. Esse resultado é esperado [3], pois as condições de contorno usadas para a velocidade intermediária $\mathbf{u}^{*}$ são as mesmas da velocidade $\mathbf{u}$. Como pode ser visto nas figuras $1 \mathrm{c}$ e $1 \mathrm{~d}$, o erro proporcional ao $\Delta t$ não é perceptível com o PI-A e LP-E e quarta ordem foi observada em todas as malhas testadas.

A figura 1e mostra a evolução do máximo do divergente discreto. No LP-A, o erro proporcional ao $\Delta t$ afeta também o divergente. No PI-A, o divergente tem um valor melhor e tende a melhorar à medida que se aproxima do estado estacionário. No entanto, o melhor valor para o divergente é obtido no LP-E. Por se tratar de um método de projeção exato, espera-se um divergente exatamente igual a zero, limitado apenas pela precisão da máquina. O observado na figura 1e está de acordo com essa expectativa.

\subsection{Vórtices de Taylor-Green}

Os vórtices de Taylor-Green são uma solução analítica para Navier-Stokes geralmente usada para medir a ordem temporal de métodos numéricos [1].

A figura 2 mostra os resultados do teste de convergência temporal. Para os esquemas PI-A e LP-E, foi obtida segunda ordem de precisão, não só para o campo de velocidade, mas também para a pressão. A maioria dos trabalhos na literatura consultada sobre métodos de projeção ou só obtém primeira ordem na pressão ou não reportam a ordem encontrada. Para o esquema LP-A, a adoção de condições de contorno simplificadas para $\mathbf{u}^{*}$ reduzem a ordem do método para primeira ordem.

\section{Conclusões}

O esquema LP-E possui a interessante característica de produzir quarta ordem de convergência espacial e segunda ordem de convergência temporal, sem a necessidade de se incluir uma aproximação para o gradiente da pressão na equação do momento e sem a necessidade de corrigir as condições de contorno para $\mathbf{u}^{*}$. Além disso, com esse esquema, a 


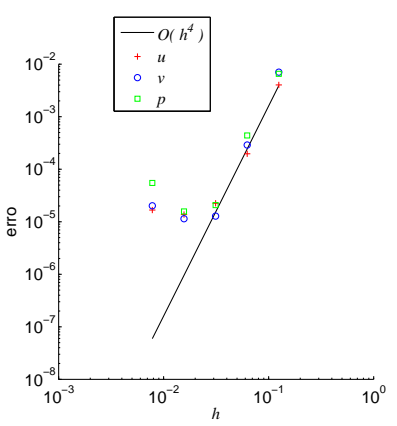

(a) LP-A com $\Delta t=10^{-4}$.

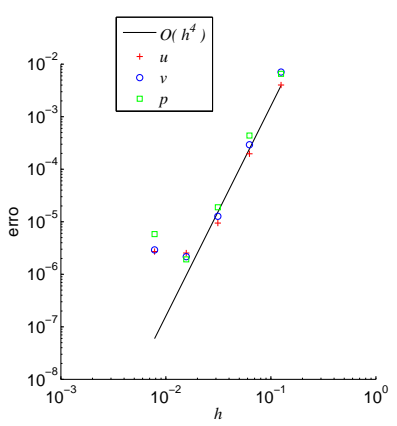

(b) LP-A com $\Delta t=10^{-5}$.

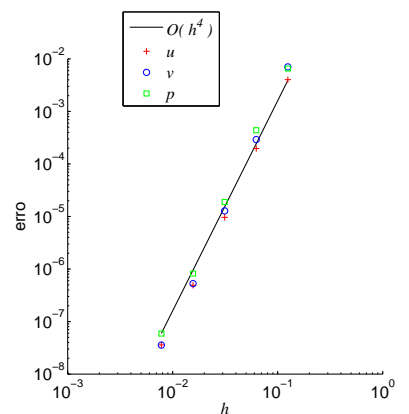

(c) PI-A.

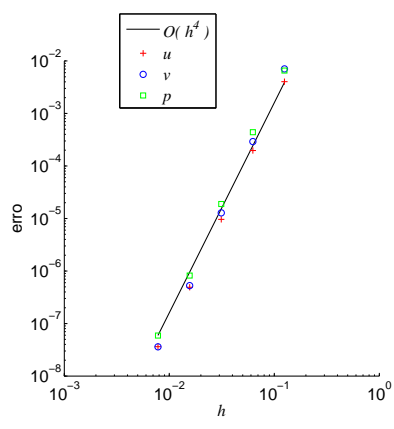

(d) LP-E.

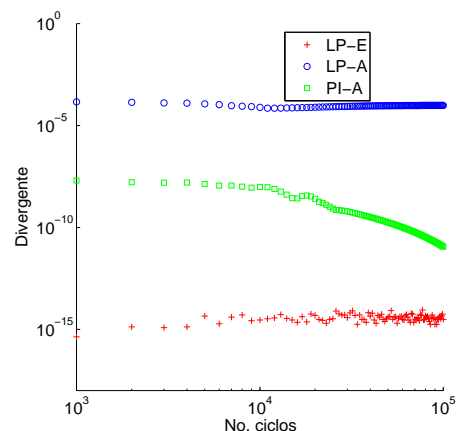

(e) LP-E.

Figura 1: Teste de convergência espacial baseado na solução analítica de estado estacionário apresentada em [8]. O erro é calculado como a diferença entre a solução numérica e a solução analítica na norma $L_{\infty}$. A linha contínua representa quarta ordem de convergência. A viscosidade cinemática usada é $\nu=10^{-2}$, o passo de tempo é $\Delta t=10^{-4} \mathrm{e}$ as malhas foram $8 \times 8,16 \times 16,32 \times 32,64 \times 64$ e $128 \times 128 . \Delta x=\Delta y=h$. Em 1e é mostrado a evolução do máximo do divergente discreto numa malha fixa $8 \times 8$.

condição de incompressibilidade é satisfeita em todos os passos de tempo de forma exata, a menos de erros de máquina.

Os resultados apresentados aqui foram publicados em Computers \& Fluids [6].

\section{Agradecimentos}

Pelo apoio financeiro, processos n²012/04471-5 e 2013/21501-8 Fundação de Amparo à Pesquisa do Estado de São Paulo (FAPESP).

\section{Referências}

[1] S. Abide, and S. Viazzo, A 2D compact fourth-order projection decomposition method, J. of Comp. Physics, (2005). DOI:10.1016/j.jcp.2004.12.005. 


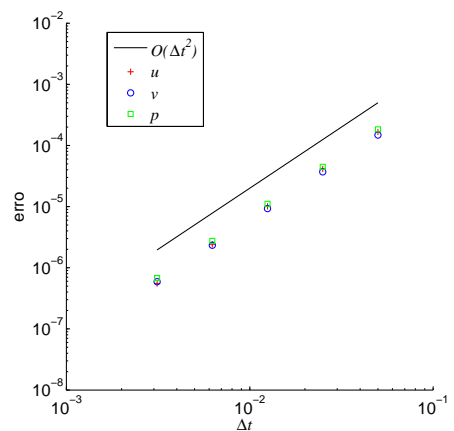

(a) PI-A.

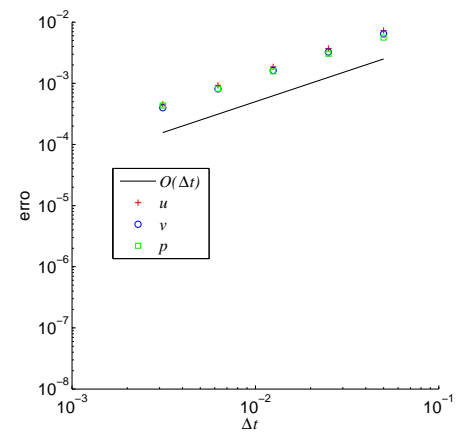

(b) LP-A.

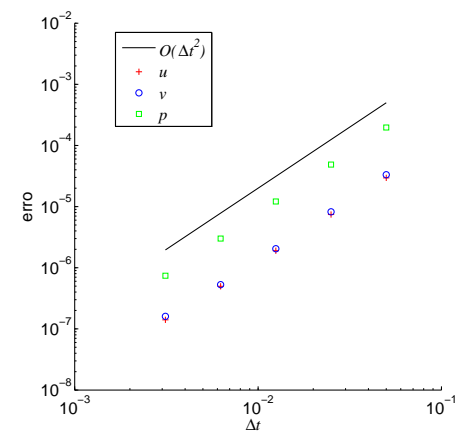

(c) LP-E.

Figura 2: Convergência temporal para o problema vórtices de Taylor-Green. A simulação foi executada até $t=1$. A viscosidade cinemática usada é $\nu=10^{-2}$. Os erros são calculados em relação a solução de referência com $\Delta t=10^{-4}$ na norma $L_{\infty}$. A malha é fixa em $20 \times 20$. Em 2a e 2c a linha contínua representa segunda ordem enquanto que em $2 \mathrm{~b}$ primeira ordem.

[2] J. B. Bell, P. Colella and H. M. Glaz, A second-order projection method for the incompressible Navier-Stokes equations, J. of Comp. Physics, (1989), DOI:10.1016/00219991(89)90151-4.

[3] D. L. Brown and R. Cortez and M. L. Minion, Accurate projection methods for the incompressible Navier-Stokes equations, J. Comp. Physics, (2001), DOI:10.1006/jcph.2001.6715.

[4] J. Kim and P. Moin, Application of a fractional-step method to incompressible NavierStokes equations, J. of Comp. Physics, (1985). DOI:10.1016/0021-9991(85)90148-2.

[5] S. K. Lele, Compact Finite Difference Schemes with Spectral-like Resolution, J. of Comp. Physics, (1992). DOI:10.1016/0021-9991(92)90324-R.

[6] G. A. Reis, I. V. M. Tasso, L. F. Souza and J. A. Cuminato, A compact finite differences exact projection method for the Navier-Stokes equations on a staggered grid with fourth-order spatial precision, Computers \& Fluids, (2015), DOI: http://dx.doi.org/10.1016/j.compfluid.2015.06.015.

[7] F. S. Sousa, C. M. Oishi and C. G. Buscaglia, Spurious transients of projection methods in microflow simulations, Computer Methods in Applied Mechanics and Engineering, (2015), DOI:10.1016/j.cma.2014.11.039.

[8] T. M. Shih, C. H. Tan and B. C. Hwang, Effects of grid staggering on numerical schemes, International Journal for Numerical Methods in Fluids, (1989), DOI: 10.1002/fld.1650090206.

[9] P. Wesseling, Principles of computational fluid dynamics, (2009), Vol. 29. Springer Science \& Business Media. 